\title{
Nanostructured Hollow Tubes Based on Chitosan and Alginate Multilayers
}

\author{
Joana M. Silva, Ana Rita C. Duarte, Catarina A. Custódio, Praveen Sher, Ana I. Neto, \\ António C. M. Pinho, Jaime Fonseca, Rui L. Reis, and João F. Mano*
}

The design and production of structures with nanometer-sized polymer films based on layer-by-layer ( $L b L$ ) are of particular interest for tissue engineering since they allow the precise control of physical and biochemical cues of implantable devices. In this work, a method is developed for the preparation of nanostructured hollow multilayers tubes combining LbL and template leaching. The aim is to produce hollow tubes based on polyelectrolyte multilayer films with tuned physical-chemical properties and study their effects on cell behavior. The final tubular structures are characterized by differential scanning calorimetry (DSC), Fourier transform infrared spectroscopy (FTIR), microscopy, swelling, and mechanical tests, including dynamic mechanical analysis (DMA) in physiological simulated conditions. It is found that more robust films could be produced upon chemical cross-linking with genipin. In particular, the mechanical properties confirms the viscoelastic properties and a storage and young modulus about two times higher. The water uptake decreases from about $390 \%$ to $110 \%$ after the cross-linking. The biological performance is assessed in terms of cell adhesion, viability, and proliferation. The results obtained with the cross-linked tubes demonstrate that these are more suitable structures for cell adhesion and spreading. The results suggest the potential of these structures to boost the development of innovative tubular structures for tissue engineering approaches.

\section{Introduction}

The fabrication of functional hybrid materials composed of nanoscale building blocks is one representative way in which nanotechnology can contribute to biology and medicine. ${ }^{[1,2]}$ Since its introduction 20 years ago by Decher and co-workers, layer-by-layer (LbL) has been attracted particular attention in this field due to its simplicity and versatility. ${ }^{[3,4]}$ The basic principle of this technique relies on the fabrication of nanostructured multilayered films by assembly and stratified organization of polyanions, polycations, or bipolar amphiphiles on the material surface. ${ }^{[5-12]}$ The driving forces behind the polyelectrolyte multilayers (PEMs) deposition include electrostatic as well as non-electrostatic interactions, such as covalent bond, hydrogen bonding, charge transfer, hydrophobic, host-guest, and coordination bond interactions. ${ }^{[3,13,14]}$ Moreover, the charge overcompensation is the key rule for PEMs formation. ${ }^{[15]}$ In a pioneering work, Schelnoff and coworkers studied the concept of intrinsic (i.e., polyelectrolytes of opposite charges) versus extrinsic charge matching (i.e., counterions), which revealed that PEMs formation is not only of electrostatic origin but also the gain in entropy due to the release of counterions, very similar to what is observed in polyelectrolyte complexes (PECs) formation. ${ }^{[14,16,17]}$ Thus, film's global electroneutrality is assured by the presence of counterions, which are displaced by oppositely charged polyelectrolytes upon complexation. ${ }^{[14]}$ The most employed LbL protocols are based on the build-up of PEMs with tunable thickness, chemical and physical properties onto flat surfaces. ${ }^{[18]}$ Nowadays, more complex structures prepared with LbL have been reported, including freestanding films, capsules, and porous scaffolds..$^{[4,19-28]}$ Porous scaffolds were obtained by coating free-packet of leachable spherical templates or by beads agglomeration. ${ }^{[23,24,28]}$ On the other side, the freestanding films and capsules can be obtained using sacrificial templates or hydrophobic templates..$^{[4,19,20,22,26,27,29]}$ These 3D structures have a great potential for tissue engineering (TE) approaches since they can be synthetized under mild conditions using a wide range of materials. ${ }^{[7,10,12]}$ These features turn LbL an excellent candidate for surface modification and for

DOI: 10.1002/adhm201300265 


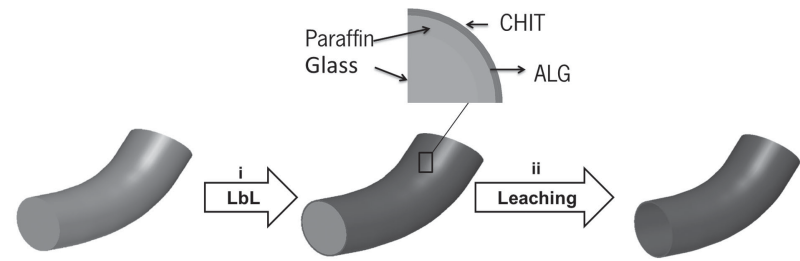

Paraffin tube

Scheme 1. Production steps of hollow tubes: i) Construction of PEMs on paraffin-coated tubes with polyelectrolytes, ALG, and CHIT (LbL); ii) Leach out the entire template using an organic solvent, such as DCM.

the creation of 2D and 3D structures with mechanical stability and adequate cell nano/microenvironments that may improve tissue regeneration.

Therefore, although the existence of a wide variety of 3D structures created by LbL, the production of hollow tubular structures with potential application for vascular TE construct is still a challenge. LbL appeared as a powerful technique since it offers a control over the chemical, mechanical, topographical, and diffusion properties of the obtained multilayers. To achieve a functional hollow tubular structure, we propose the development of tube-like elements combining LbL and leaching of sacrificial templates. A schematic representation is given in Scheme 1. Here, we report the proof-of-concept for the fabrication of hollow tubes composed by PEMs of chitosan (CHIT) and alginate (ALG). CHIT and ALG are often successfully applied in LbL due to their biocompatibility, non-cytotoxic character, and structural characteristics similar to glycosaminoglycans (GAG's). ${ }^{[15,19,21,24,27,30-32]}$ Polysaccharides PEMs often lack stability in physiological conditions, exhibiting low stiffness and poor cell adhesion due to high hydration. ${ }^{[33]}$ These drawbacks can be avoided by cross-linking. ${ }^{[34-38]}$ Genipin is a natural cross-linking agent, which has shown to be quite efficient in PEMs. ${ }^{[33,39-41]}$ The main aim of this work is to evaluate the potential of hollow tubes composed by ALG/CHIT, as well as the effect of stabilizing this system at physiological conditions by genipin cross-linking. A series of physicochemical and biological tests was performed to demonstrate the initial hypothesis.

\section{Results and Discussion}

\subsection{Nanostructured Hollow Tubes: Physico- chemical Characterization}

Differential scanning calorimetry (DSC) measurements (Figure 1A) were executed on ALG/CHIT tube as well as on paraffin powder, in order to confirm the efficient leaching of the sacrificial template. Regarding the results obtained, the paraffin powder has a perfect define melting peak at $60^{\circ} \mathrm{C} .{ }^{[42]}$ This peak was not present in the tubular structures, which indicate the successful leaching of all the paraffin used as a template.
Fourier transform infrared spectroscopy (FTIR) measurements (Figure 1B) were performed on the ALG/CHIT tube as well as on both CHIT and ALG powders in order to identify the presence of both polysaccharides in the final device. The spectra of CHIT and ALG are very similar, as expected, reflecting the similarities in the chemical structure of both materials. The two biomacromolecules share some common peaks between $950-1200 \mathrm{~cm}^{-1}$ representatives of the skeletal vibration of saccharide rings of CHIT and ALG. ${ }^{[27,32]}$ Two other regions can be observed in the ALG/CHIT tubes spectrum: the band at $1606 \mathrm{~cm}^{-1}$, attributed to -COO- asymmetric stretch from ALG and the band at $1420-1500 \mathrm{~cm}^{-1}$ corresponding to the amide II of CHIT. ${ }^{[27,32]}$ The spectrum of the tube show, globally, the absorption peaks from both CHIT and ALG, which is indicative of the presence of both raw materials in the final structure.

\subsection{Morphology}

Bottom-up approaches constitute a powerful tool to produce 3D structures, since they offer the possibility to mimic the hierarchical organization of native tissues enabling to have a control on the physicochemical properties and produce distinct cell nano/microenvironments. In this work, it has been hypothesized that a hollow tube could be prepared combining LbL with leaching of sacrificial templates. Basically, tubular templates of paraffin were coated with PEMs of ALG and CHIT. These polysaccharides were already reported to conceive a homogeneous polymeric coating using LbL. ${ }^{[32]}$ Afterwards, the sacrificial template was completely leached out. The hollow tube entirely composed of ALG/CHIT was dried using supercritical fluid technology, in order to avoid the collapse of the structure and to ensure the complete removal of the organic solvent (DCM) (Scheme 1). ${ }^{[43]}$ To increase the mechanical strength and consequently the stability, the tubes were cross-linked using genipin. The morphology of both structures was observed by scanning electron microscopy (SEM). SEM images of dried tubes reveal a noticeable hollow tube imprint of the wax-coated template used. This concept allows the production of hollow structures with controllable diameter size. The morphology of the inner and external side of both tubes (ALG/CHIT tube and crosslinked ALG/CHIT tube) is homogeneous, revealing a uniform distribution of both polysaccharides (Figure 2A,B). However, the cross-linked tubes exhibit a higher roughness. The increase

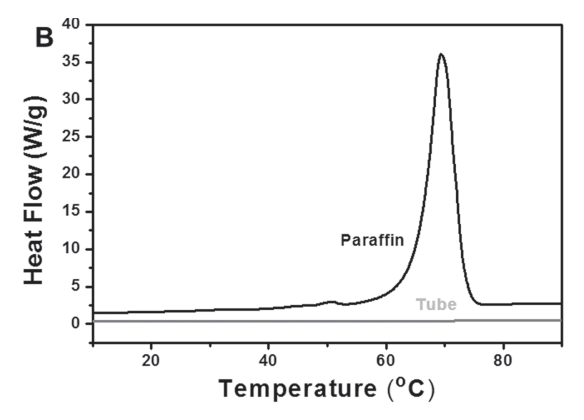

Figure 1. Physicochemical characterization of tubes: A) FTIR measurements of ALG/CHIT tube and pure polysaccharides (CHIT and ALG); B) DSC scans obtained during heating of ALG/ CHIT tube and paraffin powder.

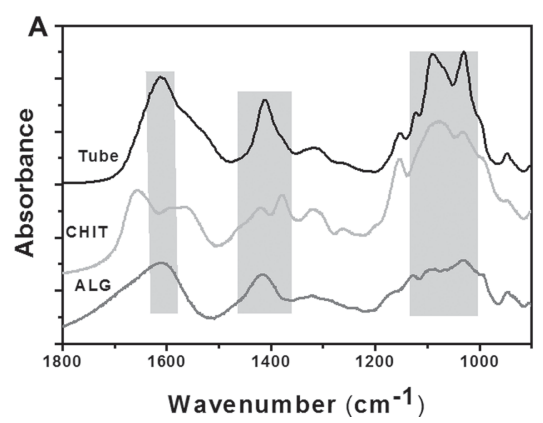



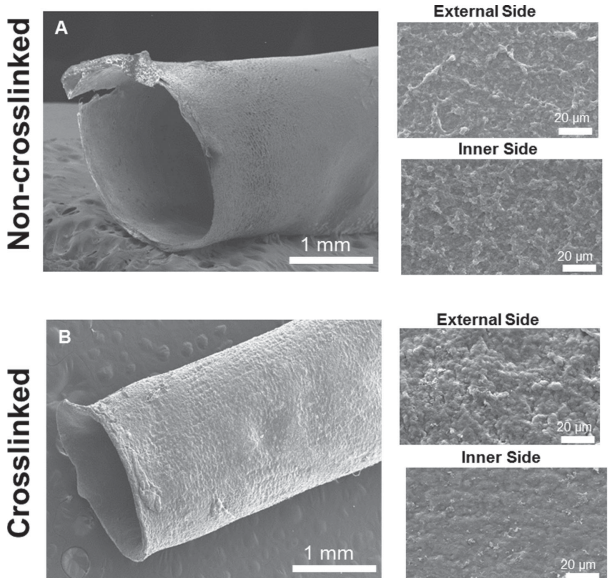
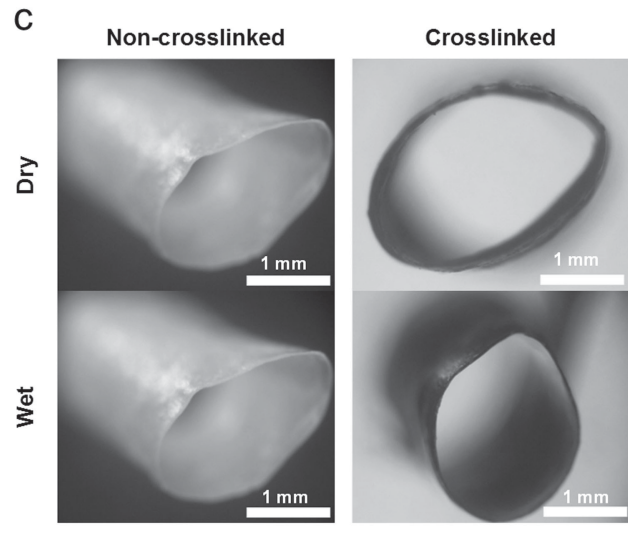

Figure 2. Tube morphology: SEM images of A) ALG/CHIT and B) crosslinked ALG/CHIT tubes. C) Optical microscopy image of both formulations in dry and wet state.

of roughness by genipin cross-linking was already reported before. ${ }^{[41]}$ Further structural information in physiological-like conditions was obtained by optical microscopy (Figure 2C). Both structures maintained the tubular structure in wet conditions, as the water uptake is higher (see next section). ${ }^{[4]}$ However, the results clearly reveal a more stable and handling structure of cross-linked ALG/CHIT tubes. This behavior is even more pronounced in an aqueous environment.

\subsection{Swelling Ability}

The developed hollow tubular structure is composed by polyelectrolytes with abundant hydrophilic groups, such as hydroxyl, amino, and carboxyl groups, which can promote water-uptake. ${ }^{[17,45,46]}$ The swelling ability was evaluated in Dulbecco's modified eagle medium (DMEM) at $37{ }^{\circ} \mathrm{C}$ during $21 \mathrm{~d}$ using freestanding films (Figure 3). Using these flat templates, it is possible to avoid the overestimating of water-uptake that could occur due to the water entrapment in the void space of the tubes. The results show that the water uptake increases mainly during the first $15 \mathrm{~min}$ and then tends to remain stable, reaching the equilibrium. The same trend is observed in both samples (with or without cross-linking). As expected, ALG/CHIT films have a higher water uptake $(\approx 390 \%)$ when
A

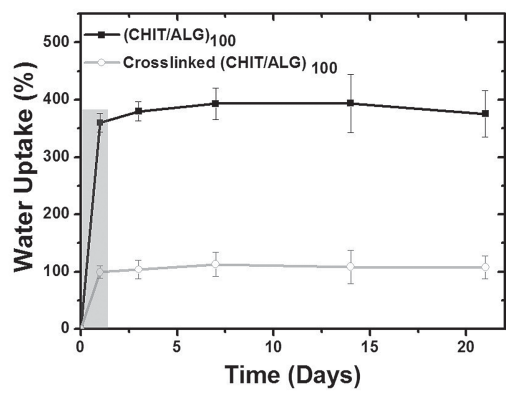

B

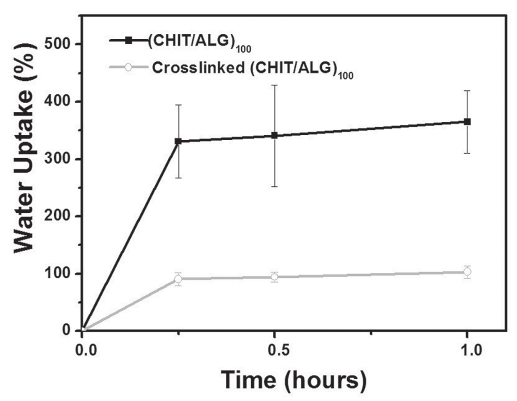

Figure 3. Swelling ability of ALG/CHIT tubes with or without cross-linking in DMEM at $37^{\circ} \mathrm{C}$ : for A) $21 \mathrm{~d}$ and B) $1 \mathrm{~h}$. compared with the cross-linked ALG/CHIT ones ( $\approx 110 \%)$. This is consistent with the results obtained in terms of thickness variation. ALG/CHIT films show $2.46 \pm 0.189$ fold increase in thickness whereas the cross-linked ALG/CHIT films $1.2943 \pm$ 0.0068 fold increase.

\subsection{Mechanical Properties}

The mechanical and viscoelastic properties of an implantable device are fundamental for its performance in vivo. ${ }^{[47]}$ Dynamical mechanical analysis (DMA) is an adequate nondestructive tool for characterizing biomaterials in test conditions that can more closely simulate the physiological environment. ${ }^{[48-50]}$ DMA experiments were performed in a hydrated environment and at $37{ }^{\circ} \mathrm{C}$, allowing the assessment of the mechanical properties in more realistic conditions. ${ }^{[47]}$ The storage modulus $\left(E^{\prime}\right)$ and loss factor $(\tan \delta$ ) as a function of frequency of the developed tubes are presented in Figure 4A,B. The results show a slight increase in both $E^{\prime}$ and $\tan \delta$ with increasing frequency. Moreover, the $E^{\prime}$ of cross-linked ALG/CHIT tubes is significantly higher than in the native tubes, showing the effective stiffening effect provided by the genipin treatment. In both cases, no evident variation of $E^{\prime}$ along the frequency axis is seen, indicating that no relaxation phenomena took place in the tubes within the time scale covered by the experiments. The $\tan \delta$ is the ratio between the energy lost by viscous mechanisms and the energy stored in the elastic component. ${ }^{[49,50]}$ Comparing the $\tan \delta$ of both formulations, ranging from 0.1 and 0.3 , the values confirm the viscoelastic behavior of both tubular structures.

The mechanical properties of these structures were also evaluated in dry state using an universal mechanical testing equipment (tensile mode). Figure 4C shows representative stress-strain curves for both formulations. In the native tubes, the modulus 


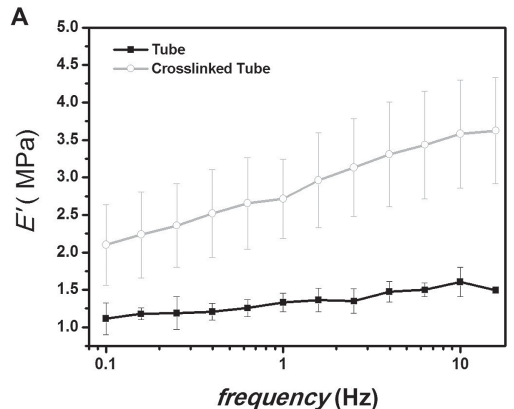

C

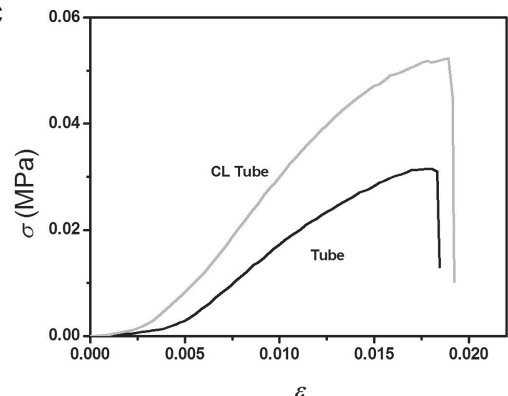

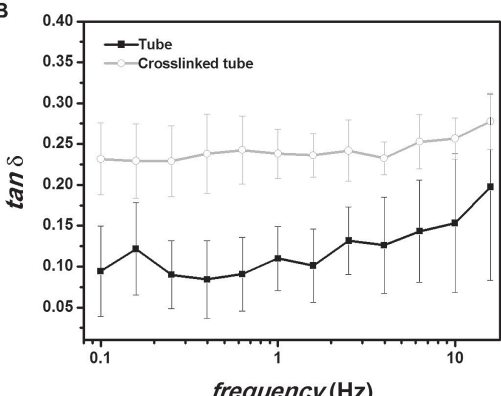

D

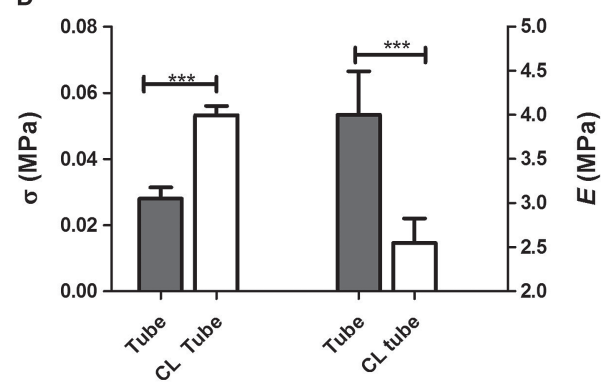

Figure 4. Mechanical properties: Variations of A) Storage modulus $\left(E^{\prime}\right)$ and B) loss factor (tan $\delta$ ) of ALG/CHIT tubes with or without cross-linking obtained by DMA measurements in wet conditions $\left(37^{\circ} \mathrm{C}\right)$. C) Strain-stress curves for ALG/CHIT tubes ("tubes") and cross-linked ALG/CHIT tubes ("CL tubes"). D) Ultimate tensile strength $(\sigma)$ and Young modulus $(E)$ of ALC/CHIT tubes ("tubes") and cross-linked ALG/CHIT tubes ("CL tubes"). Significant differences between each were found for $* * * p<0.001$.

values are two times higher when compared with the wet state (see Figure 4D). This is consistent with the higher water uptake ability of the native tubes and the plasticization effect of water molecules in such kind of polysaccharides increasing their molecular mobility and decreasing the stiffness of the material. ${ }^{[48]}$ Moreover, the ultimate strength is also higher on

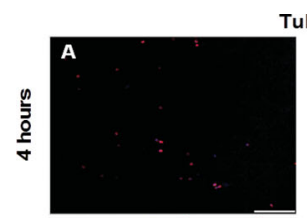

Tube

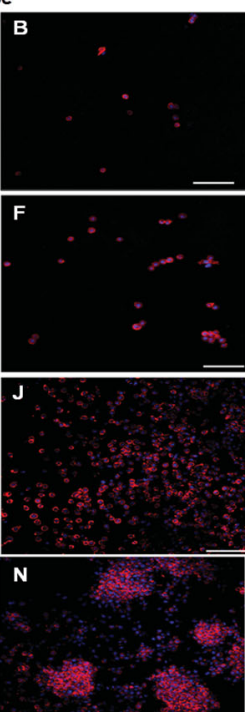

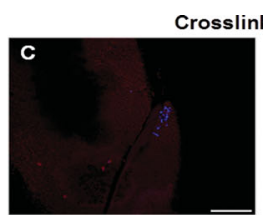
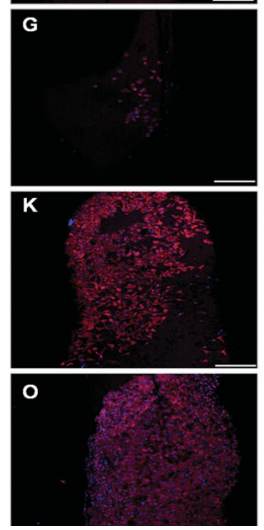

cross-linked tubes (see Figure 4D). All together these results confirm the hypothesis that cross-linking of PEMs with genipin substantial increases the mechanical performance of the tubular structure. It is expectable that using higher numbers of layers one could further improve the mechanical properties. The changing of the number of layers could be also used to control the transport properties across the membrane, enabling to tailor the perme-

ability of nutrients and other molecules.

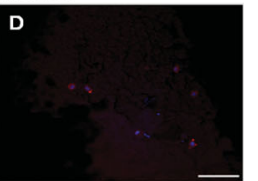

H
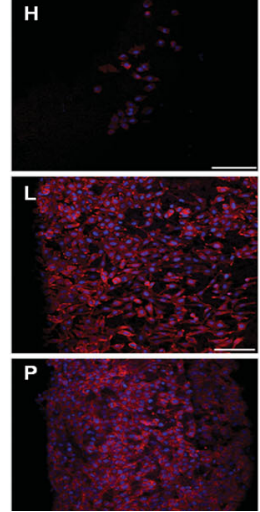

Figure 5. DAPI-phalloidin fluorescence assay at $4 \mathrm{~h}, 1,3$, and $7 \mathrm{~d}$ of culture. ALG/CHIT and cross-linked ALG/CHIT tubes. Cells nuclei were stained blue by DAPI and F-actin filaments in red by phalloidin. Scale bar represents $200 \mu \mathrm{m}$ and $100 \mu \mathrm{m}$ in lower and higher magnification images, respectively.

\subsection{Biological Performance}

L929 cells were grown on native and crosslinked tubes made of 100 bilayers of ALG and CHIT. The cell adhesion and morphology were studied by DAPI-phalloidin (Figure 5). After $4 \mathrm{~h}$, the cells in suspension start to adhere on the surface and behave quite different on both formulations. This tendency continues after $1 \mathrm{~d}$ and is even more noticeable after 3 and $7 \mathrm{~d}$ in culture. The cells seeded on cross-linked tubes are well spread and anchored to the PEM film. On the other hand, the cells cultured on the native films are less susceptible to create strong anchors, which can occur due to the softness, high hydration, and easy deformation of these films. Cell morphology was further studied by SEM and the results were concordant with DAPI-phalloidin assay (see 


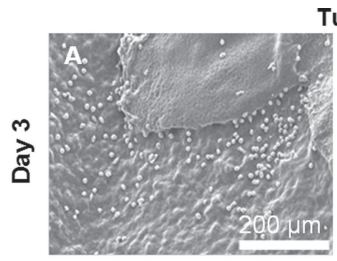

Tube

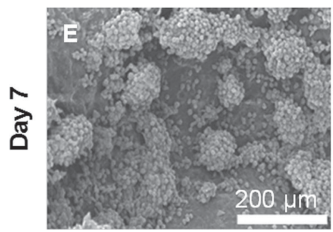

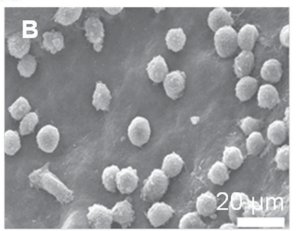

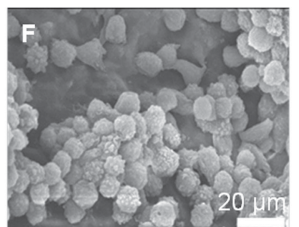

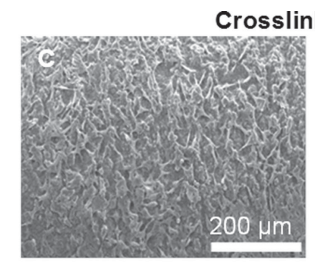

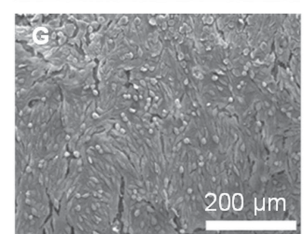

Crosslinked Tube
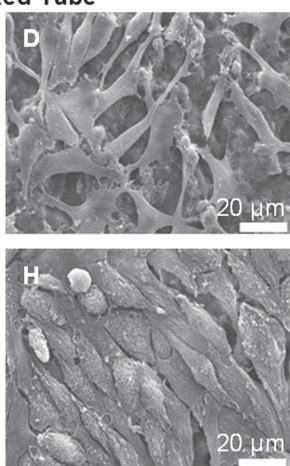

Figure 6. SEM images of L929 seeded on ALG/CHIT and cross-linked ALG/CHIT tubes at day 3 and day 7 of culture.

images in Figure 6). The results show that after $3 \mathrm{~d}$ the cells seeded on cross-linked tubes are more spread out along the tube and present a stretched morphology. On the other side, the cells seeded on native tubes tend to aggregated, presenting a round morphology. The same tendency is evidenced after $7 \mathrm{~d}$. These results are expectable since the cross-linking reduces the hydration of the film and increase the stiffness, which plays a role in many cellular processes ranging from motility, to spreading and differentiation. ${ }^{[37,51]}$

Regarding the cell viability assessment, in general, the results reveal that cells are able to remain viable in the tubes up to $7 \mathrm{~d}$ in culture (Figure 7A). MTS results show a statistically significant enhancement of total cell viability for crosslinked tubes for 3 and $7 \mathrm{~d}$ of culture when compared with native tubes. This is a consequence of better cell adherence and spreading on cross-linked tubes and it is corroborated with other studies reported in literature for other types of cells

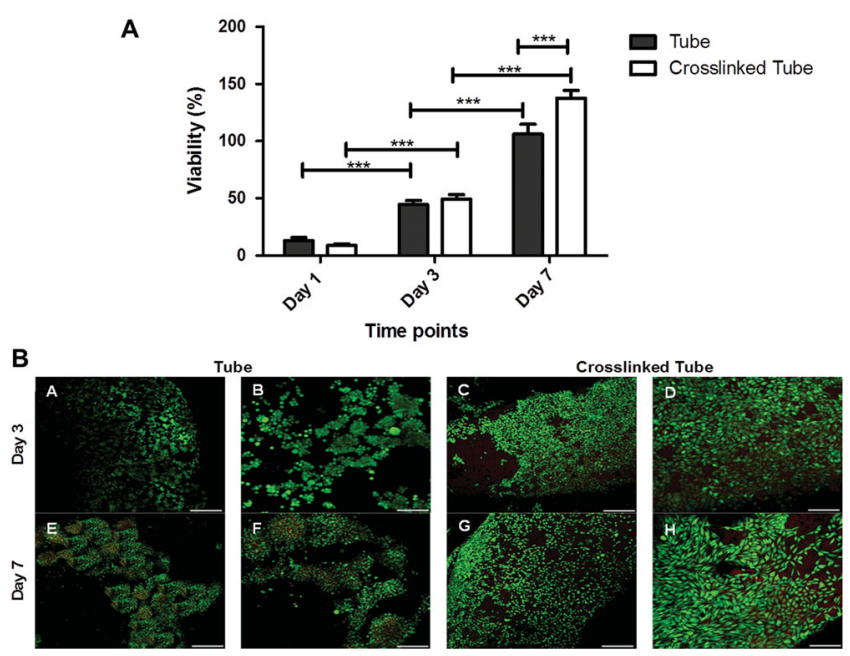

Figure 7. Cell viability at 1,3 , or $7 \mathrm{~d}$ of culture on ALG/CHIT and crosslinked ALG/CHIT tubes: A) MTS assay. Statistical differences in grouped by time point analysis are marked with (**) and (***), which stand for $p<0.01$ and $p<0.001$, respectively; B) Live-dead fluorescence assay. Living cells were stained green by calcein and dead cells red by propidium iodide. Scale bar represents $200 \mu \mathrm{m}$ and $100 \mu \mathrm{m}$ in lower and higher magnification images, respectively. (fibroblasts, smooth muscle cells, and neurons) using different PEMs. ${ }^{[18,33-38,41,52,53]}$ This behavior is in agreement with the results obtained during live/dead assay (Figure 7B). Almost no dead cells are observed on cross-linked films when compared with the native films. The cells on native films grown in aggregates and they were not well spread along the surface. Furthermore, the cell proliferation was also assessed as a function of culture time up to $7 \mathrm{~d}$ by DNA quantification (Figure 8). The results obtained for native ALG/CHIT tubes and cross-linked ALG/CHIT tubes show that the number of cells increases in both types of tubes with increasing culture time. Significant differences were observed between the two formulations after $3 \mathrm{~d}$ and the differences were even more pronounced after $7 \mathrm{~d}$ in culture. All together, the results demonstrate the important role of film mechanics in cell adhesive properties.

\section{Conclusion}

Nanostructured hollow tubes of ALG and CHIT were successfully obtained using LbL and template leaching. The hollow tubes were subsequently cross-linked with genipin, leading to a decrease of water uptake and improvement of mechanical properties. The results suggested that these properties affected cell behavior regarding their adhesion, metabolic activity, and proliferation. We believe that the knowledge generated by this research will open new perspectives in the development of

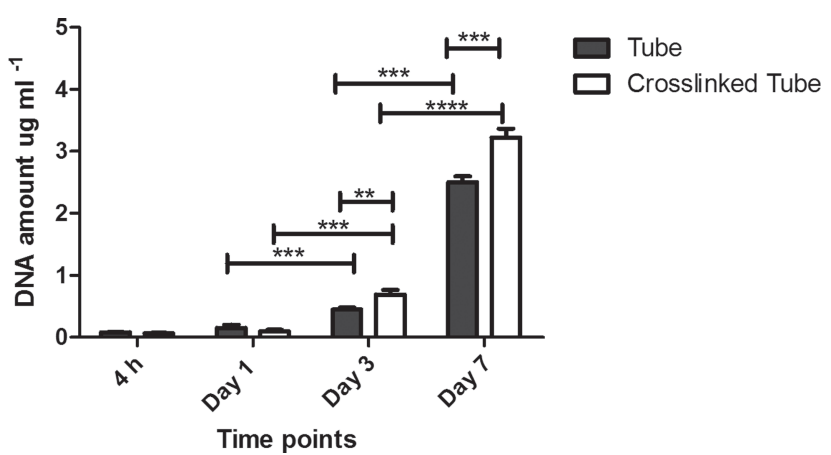

Figure 8. DNA assay on ALG/CHIT and cross-linked ALG/CHIT tubes seeded with L929. Significant differences between each cell type at different time points were found for $p<0.01(*)$ and $p<0.001$ ( $(* * * *)$. 
hybrid devices for vascular TE approaches. The most promising feature of the proposed multilayered tubes is the ability to tune different properties in one structure, creating novel multifunctional materials.

\section{Experimental Section}

Production of Tube-Like Elements: The templates were produced by dip-coating glass tubes $(\varnothing 1 \mathrm{~mm}$ ) in molten paraffin (Jojoba Desert Whale, USA). Upon solidification, an homogeneous coating of paraffin (thickness $\approx 600 \mu \mathrm{m}$ ) was produced around the glass tube. The two polyelectrolytes used to process the multilayers were CHIT medium molecular weight $\left(\bar{M}_{\mathrm{w}}=190.000-310.000 \mathrm{Da}\right.$, $88 \%$ degree of deacetylation, Sigma Aldrich, USA) and low viscosity ALG $(\approx 250 \mathrm{cp}$, Sigma Aldrich, USA). The polyelectrolyte solutions were freshly prepared $(0.2 \%, \mathrm{w} / \mathrm{v})$. For the adjustment of $\mathrm{pH}$, an sodium acetate buffer $(0.1 \mathrm{M})$ was prepared at $\mathrm{pH} 5.5$ in the presence of additional salt $(0.15 \mathrm{M}$ $\mathrm{NaCl}, \mathrm{pH}$ 5.5). The CHIT solution was injected standing for $8 \mathrm{~min}$ to allow the adsorption equilibrium at the tubular template surface. After rinsing with sodium acetate buffer $/ 0.15 \mathrm{M} \mathrm{NaCl}$ (two times $2 \mathrm{~min}$ ), the same procedure was followed for the deposition of ALG. The steps were repeated to the desire number of layer (100 double layers $(\mathrm{dL})$ ), using an in-house developed dipping robot. The coated structures were placed in dichloromethane (DCM, Sigma Aldrich, USA) to leach out the paraffin layer present between the glass substrate and the PEMs. DCM was changed every day. After leaching, the structures were dried using supercritical carbon dioxide. ${ }^{[43]}$ The samples were loaded in a high pressure vessel heated at $37{ }^{\circ} \mathrm{C}$ and pressurized to 80 bar. A carbon dioxide stream was passed through the vessel at constant rate for $1 \mathrm{~h}$ to ensure complete removal of the organic solvent. After dried, some of the tubes were cross-linked, with genipin (Wako chemical, USA). A genipin solution $\left(5 \mathrm{mg} \mathrm{mL}^{-1}\right)$ was prepared by dissolving the adequate amount of lyophilized genipin into a dimethyl sulfoxide (Sigma Aldrich, USA)/sodium acetate buffer $(0.15 \mathrm{M} \mathrm{NaCl}, \mathrm{pH} 5.5)$ mixture $(1: 4, \mathrm{v} / \mathrm{v})$. The cross-linking agent solution was incubated with the tubes overnight. Afterwards, the tubes were extensively washed in absolute ethanol and dried at $37^{\circ} \mathrm{C}$

Production of Freestanding Multilayer Films: Freestanding multilayer films were produced using a polypropylene support, following a protocol previously used. [27] The supports were coated using ALG/CHIT PEMs, precisely in the same conditions of the tubes. After dried, the membranes could be easily detached from the substrate.

Morphology: The morphology of the tubes was observed by SEM, using a Leica Cambridge S 360 (UK) operated at $15 \mathrm{kV}$ accelerating voltage. All the samples were sputtered with a conductive gold layer, using a sputter coater SC502 (Fison instruments, UK). The morphology of tubes in wet state was also evaluated using a stereomicroscope (Zeiss, Germany) with a color camera (Nikon G12).

Differential Scanning Calorimetry: The DSC experiments were performed in a TA instrument DSC Q100 model (Thermal analysis \& analyzers, USA), using paraffin powder and ALG/CHIT tubes (with 5-5.5 mg sample $\mathrm{wt}$ ). The temperature program included a step of heating from $10^{\circ} \mathrm{C}$ to $100^{\circ} \mathrm{C}$, an isothermal step of 2 min followed from cooling to $0{ }^{\circ} \mathrm{C}$ and a new heating step to $100{ }^{\circ} \mathrm{C}$. Only the data of the last heating step were registered. All measurements were performed under a nitrogen atmosphere (purge gas flux of ca. $50 \mathrm{~mL} \mathrm{~min}$ ) and $10^{\circ} \mathrm{C} \mathrm{min}^{-1}$ heating rate.

Fourier Transform Infrared (FTIR) Spectroscopy: FTIR measurements were recorded using an IR-Prestige-21 spectrophotometer (Shimadzu Scientific Instruments, USA) by averaging 34 individual scans over the range from $4400 \mathrm{~cm}^{-1}$ to $400 \mathrm{~cm}^{-1}$. The samples (CHIT powder, ALG powder and tube) were prepared in potassium bromide discs.

Swelling Test: The water-uptake ability of the PEMs was measured using freestanding films. Dry films with known weight were soaked in DMEM (Sigma, USA) at $\mathrm{pH} 7.4$ up to $21 \mathrm{~d}$ at $37^{\circ} \mathrm{C}$. The swollen films were removed at predetermined time points $(t=15 \mathrm{~min}, 30 \mathrm{~min}, 1 \mathrm{~h}$,
$1 \mathrm{~d}, 3 \mathrm{~d}, 7 \mathrm{~d}, 14 \mathrm{~d}$, and $21 \mathrm{~d}$ ). After removing the excess of DMEM using a filter paper (Filter Lab, Spain), the freestanding films were weighed with an analytical balance (Denver Instrument, Germany). The water uptake was calculated as followed:

Water uptake $\%=\frac{w_{\mathrm{w}}-w_{\mathrm{d}}}{w_{\mathrm{d}}} \times 100$

where $w_{\mathrm{w}}$ and $w_{\mathrm{d}}$ are the weights of swollen and dried freestanding films, respectively.

During the first hour, the freestanding films were also used to estimate the thickness after immersion in DMEM, using a digital micrometer with a precision of $0.001 \mathrm{~mm}$.

Mechanical Tests: The dynamic mechanical analysis measurements were performed using a TRITEC8000B DMA (Trinton Technology, UK). Tubes were immersed in phosphate buffered saline (PBS) until equilibrium was reached (minimum $10 \mathrm{~min}$, according to the swelling tests). Samples were tested at physiological-like conditions, that is, immersed in PBS at $37{ }^{\circ} \mathrm{C}$ placed in a Teflon reservoir. After measuring the geometry, the samples were clamped in the DMA apparatus and immersed in the PBS bath. Tensile experiments were carried out following cycles of increasing frequency ranging from 0.1 to $20 \mathrm{~Hz}$, with constant strain amplitude of $20 \mu \mathrm{m}$. Three samples were used per condition. The mechanical properties of tubes were also studied in an universal mechanical testing machine (Instron model 5540, USA) according to the ASTMC749-08 standard method, where the samples were fixed in each extremity. A cross-head speed of $5 \mathrm{~mm} \mathrm{~min} \mathrm{mis}^{-1}$ was employed and the samples were stressed until enough force was applied to trigger their rupture. Strain-stress curves were obtained for each experiment. The initial linear region of stress-strain curves was used to determine the Young modulus by the tangent method. Five samples were tested per condition.

Cell Culture: To evaluate the biological performance of ALG/CHIT and cross-linked ALG/CHIT tubes, cell culture studies were performed with L929, a mouse fibroblast of connective tissue cell line (European Collection of Cell Cultures (ECCC), UK). Cells were cultured in DMEM (Sigma, USA), supplemented by $10 \%$ heat-inactivated fetal bovine serum (FBS; Biochrom AG, Germany) and 1\% antibiotic- antimicotic (Gibco, USA).

Morphological Characterization-DAPI-Phalloidin and Scanning Electron Microscopy: Seeded ALG/CHIT and cross-linked ALG/CHIT tubes were incubated for $4 \mathrm{~h}, 1,3$, and $7 \mathrm{~d}$ at $37^{\circ} \mathrm{C}$ in a humidified $5 \% \mathrm{CO}_{2}$ atmosphere. 4,6-Diaminidino-2-phenylindole-dilactate (DAPI, $20 \mathrm{mg} \mathrm{mL}^{-1}$, Sigma-Aldrich, USA) and phalloidintetramethylrhodamine $B$ isothiocyanate dyes (phalloidin, $10 \mathrm{mg} \mathrm{mL}^{-1}$, Sigma-Aldrich, USA) were used to perform a DAPI-phalloidin assay ( $n=5$ samples per well, in triplicate). Briefly at each timepoint, culture medium was removed and the samples fixed in 10\% formalin. After $1 \mathrm{~h}$, formalin was removed and replaced by PBS. Upon PBS washing, $1 \mathrm{~mL}$ of PBS containing $10 \mu \mathrm{L}$ of phalloidin was added for $40 \mathrm{~min}$ at room temperature and protected from light. After extensively washing with PBS, samples were stained with DAPI by adding $10 \mu \mathrm{L}$ of it in $1 \mathrm{~mL}$ of PBS for $10 \mathrm{~min}$. After DAPI staining samples were washed three times with PBS and visualized in the dark by confocal laser microscopy. In both assays, double staining was obtained in Z-stack mode with a resolution of $4.5 \mu \mathrm{m}(10 x)$ and $2 \mu \mathrm{m}(20 \times)$ between slides by using the Olympus Fluoview 3.1 software version. For SEM analysis, samples were dehydrated, sputtered coated with gold, and visualized by SEM Leica Cambridge S 360 .

Cellular Viability Assay: Cell viability was assessed after each time point using the live/dead assay (calcein AM/propidium iodide (PI) staining) ( $n=5$ samples per well, in triplicate). Briefly, the tubes were incubated for $10 \mathrm{~min}$ with $2 \mu \mathrm{L}$ calcein $\mathrm{AM}\left(1 \mathrm{mg} \mathrm{mL}^{-1}\right.$, Molecular

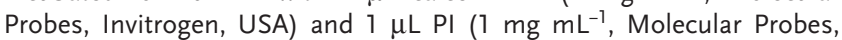
Invitrogen, USA) in $1 \mathrm{~mL}$ PBS protected from light. The tubes were washed with PBS to remove residual fluorescent and visualized by confocal laser microscopy (FV1000, Olympus, Germany). The viability of cells seeded on tubes was also tested using a MTS colorimetric assay (Cell Titer 96 AQueous One Solution Cell Proliferation Assay, Promega, 
USA) at 1, 3, and $7 \mathrm{~d}$ of culture. Briefly, samples were washed with PBS and immersed in a mixture consisting of serum-free cell culture medium and MTS reagent in a 5:1 ratio and incubated for $3 \mathrm{~h}$ at $37^{\circ} \mathrm{C}$ in a humidified atmosphere containing $5 \% \mathrm{CO}_{2}$. After this, $100 \mu \mathrm{L}$ of each well (in triplicate) was transferred to a 96 -well plate. The amount of formazan product was measured by absorbance at a wavelength of $490 \mathrm{~nm}$ using a microplate spectrophotometer (Synergy HT, Bio-TEK, USA). The background was corrected by subtracting the absorbance obtained from tubes without cells to those with cells.

DNA Assay: DNA content was quantified using the PicoGreen DNA kit (Molecular Probes) according to the instructions from the manufacturer. Prior to DNA quantification, cells were lysed by osmotic and thermal shock and the supernatant was used for the assay. Triplicates were performed for each sample or assay standard. The plates were read on a microplate reader (BioTek, USA) using 485 and $528 \mathrm{~nm}$ as excitation and emission wavelengths, respectively, according to the spectroscopic properties of the dye. The DNA amounts were calculated using a calibration curve.

Statistical Analysis: The experiences were carried out in triplicate otherwise specified. The results were presented as mean \pm standard deviation (SD). Statistical analysis was performed by Shapiro Wilk normality test using Graph Pad Prism 5.0 for Windows. After this analysis, non-parametric (Mann-Whitney test) or parametric tests (one way anova followed by Bonferroni's test) were used depending if the samples were from normally distributed populations or not, respectively.

\section{Acknowledgements}

The authors acknowledge the financial support by the Portuguese Foundation for Science and Technology (FCT) through the doctoral and post-doctoral grants with the reference numbers SFRH/BD/81372/2011 (J.M.S.), SFRH/BD/61390/2009 (C.A.C.), SFRH/BD/73119/2010 (I.N.), and SFRH/BPD/489948/2008 (P.S.), respectively, co-financed by the Operational Human Potential Program (POPH) developed under the scope of the National Strategic Reference Framework (QREN) from the European Social Fund (FSE). The authors would also like to acknowledge the project novel smart and biomimetic materials for innovative regenerative medicine approaches (Ref.: RL1 - ABMR - NORTE-01-0124-FEDER-000016) co-financed by North Portuga Regional Operational Programme (ON.2 - O Novo Norte), under the National Strategic Reference Framework (NSRF), through the European Regional Development Fund (ERDF).

Received: July 1, 2013 Revised: July 30, 2013 Published online:

[1] P. R. Gil, L. L. del Mercato, P. del-Pino, A. Muñoz-Javier, W. J. Parak, Nano Today 2008, 3, 12.

[2] L. Moroni, J. H. Elisseeff, Mater. Today 2008, 11, 44.

[3] Z. Tang, Y. Wang, P. Podsiadlo, N. A. Kotov, Adv. Mater. 2006, 18, 3203.

[4] C. R. Correia, R. L. Reis, J. F. Mano, Biomacromolecules 2013, 14, 743.

[5] K. Ariga, J. P. Hill, Q. Ji, Phys. Chem. Chem. Phys. 2007, 9, 2319.

[6] T. Boudou, T. Crouzier, C. Nicolas, K. Ren, C. Picart, Macromol. Biosci. 2011, 11, 77.

[7] V. Gribova, R. Auzely-Velty, C. Picart, Chem. Mater. 2011, 24, 854.

[8] P. T. Hammond, AlChE J. 2011, 57, 2928.

[9] C. Jiang, V. V. Tsukruk, Adv. Mater. 2006, 18, 829.

[10] C. Picart, Curr. Med. Chem. 2008, 15, 685

[11] B. M. Wohl, J. F. J. Engbersen, J. Controlled Release 2012, 158, 2.

[12] C. Detzel, A. Larkin, P. Rajagopalan, Tissue Eng. Part B: Rev. 2011, $17,101$.
[13] M. Matsusaki, H. Ajiro, T. Kida, T. Serizawa, M. Akashi, Adv. Mater. 2012, 24, 454

[14] T. Boudou, T. Crouzier, K. Ren, G. Blin, C. Picart, Adv. Mater. 2010 22, 441.

[15] G. V. Martins, J. F. Mano, N. M. Alves, Carbohydr. Polym. 2010, 80, 570 .

[16] J. Schlenoff, H. Ly, M. Li, J. Am. Chem. Soc. 1998, 120, 7626.

[17] T. Crouzier, C. Picart, Biomacromolecules 2009, 10, 433.

[18] J. Almodovar, T. Crouzier, S. Selimovic, T. Boudou, A. Khademhosseini, C. Picart, Lab Chip 2013, 13, 1562.

[19] C. R. Correia, P. Sher, R. L. Reis, J. F. Mano, Soft Matter 2013, 9, 2125.

[20] N. L. Costa, P. Sher, J. F. Mano, Adv. Eng. Mater. 2011, 13, B218.

[21] J. R. Grech, J. Mano, R. Reis, J. Mater. Sci., Mater. Med. 2010, 21, 1855.

[22] A. L. Larkin, R. M. Davis, P. Rajagopalan, Biomacromolecules 2010, 11, 2788.

[23] E. Miranda, T. Silva, R. Reis, J. Mano, Tissue Eng. Part A 2011, 17, 2663.

[24] P. Sher, C. A. Custódio, J. F. Mano, Small 2010, 6, 2644.

[25] H. Zhu, J. Ji, J. Shen, Biomacromolecules 2004, 5, 1933.

[26] A. Szarpak, D. Cui, F. Dubreuil, B. G. De Geest, L. J. De Cock, C. Picart, R. Auzély-Velty, Biomacromolecules 2010, 11, 713.

[27] S. G. Caridade, C. Monge, F. Gilde, T. Boudou, J. F. Mano, C. Picart Biomacromolecules 2013, 14, 1653.

[28] J. M. Silva, N. Georgi, R. Costa, P. Sher, R. L. Reis, C. A. Van Blitterswijk, M. Karperien, J. F. Mano, PLoS One 2013, 8, e55451.

[29] L. Shen, J. Fu, K. Fu, C. Picart, J. Ji, Langmuir 2010, 26, 16634.

[30] G. V. Martins, E. G. Merino, J. F. Mano, N. M. Alves, Macromol. Biosci. 2010, 10, 1444.

[31] L. Richert, P. Lavalle, E. Payan, X. Z. Shu, G. D. Prestwich, J.-F. Stoltz, P. Schaaf, J.-C. Voegel, C. Picart, Langmuir 2003, 20, 448.

[32] N. M. Alves, C. Picart, J. F. Mano, Macromol. Biosci. 2009, 9, 776.

[33] C. Chaubaroux, E. Vrana, C. Debry, P. Schaaf, B. Senger, J.-C. Voegel, Y. Haikel, C. Ringwald, J. Hemmerlé, P. Lavalle, F. Boulmedais, Biomacromolecules 2012, 13, 2128.

[34] K. Ren, T. Crouzier, C. Roy, C. Picart, Adv. Funct. Mater. 2008, 18 1378.

[35] L. Richert, A. J. Engler, D. E. Discher, C. Picart, Biomacromolecules 2004, 5, 1908

[36] L. Richert, A. Schneider, D. Vautier, C. Vodouhe, N. Jessel, E. Payan, P. Schaaf, J.-C. Voegel, C. Picart, Cell Biochem. Biophys. 2006, 44, 273.

[37] A. Schneider, G. Francius, R. Obeid, P. Schwinté, J. Hemmerlé, B. Frisch, P. Schaaf, J.-C. Voegel, B. Senger, C. Picart, Langmuir 2005, 22, 1193

[38] A. Schneider, C. Vodouhê, L. Richert, G. Francius, E. Le Guen, P. Schaaf, J.-C. Voegel, B. Frisch, C. Picart, Biomacromolecules 2006, $8,139$.

[39] H. Chen, W. Ouyang, B. Lawuyi, S. Prakash, Biomacromolecules 2006, 7, 2091

[40] M. J. Moura, H. Faneca, M. P. Lima, M. H. Gil, M. M. Figueiredo, Biomacromolecules 2011, 12, 3275.

[41] A. L. Hillberg, C. A. Holmes, M. Tabrizian, Biomaterials 2009, 30, 4463.

[42] B. Li, T. Liu, L. Hu, Y. Wang, L. Gao, ACS Sustainable Chem. Eng. 2013, 1, 374.

[43] S. S. Silva, A. R. C. Duarte, A. P. Carvalho, J. F. Mano, R. L. Reis, Acta Biomater. 2011, 7, 1166.

[44] S. S. Silva, A. Motta, M. T. Rodrigues, A. F. M. Pinheiro, M. E. Gomes, J. F. Mano, R. L. Reis, C. Migliaresi, Biomacromolecules 2008, 9, 2764. 
[45] T. Crouzier, T. Boudou, C. Picart, Curr. Opin. Colloid Interface Sci. 2010, 15, 417.

[46] J. Almodovar, L. W. Place, J. Gogolski, K. Erickson, M. J. Kipper, Biomacromolecules 2011, 12, 2755.

[47] J. M. Sobral, S. G. Caridade, R. A. Sousa, J. F. Mano, R. L. Reis, Acta Biomater. 2011, 7, 1009.

[48] J. F. Mano, Macromol. Biosci. 2008, 8, 69

[49] L.-P. Yan, J. M. Oliveira, A. L. Oliveira, S. G. Caridade, J. F. Mano, R. L. Reis, Acta Biomater. 2012, 8, 289.
[50] L.-P. Yan, Y.-J. Wang, L. Ren, G. Wu, S. G. Caridade, J.-B. Fan, L.-Y. Wang, P.-H. Ji, J. M. Oliveira, J. T. Oliveira, J. F. Mano, R. L. Reis, J. Biomed. Mater. Res. Part A 2010, 95A, 465.

[51] L. Richert, F. Boulmedais, P. Lavalle, J. Mutterer, E. Ferreux, G. Decher, P. Schaaf, J.-C. Voegel, C. Picart, Biomacromolecules 2003, 5, 284.

[52] J. A. Phelps, S. Morisse, M. Hindie, M.-C. Degat, E. Pauthe, P. R. Van Tassel, Langmuir 2010, 27, 1123.

[53] O. Semenov, A. Malek, A. Bittermann, J. Vörös, A. Zisch, Tissue Eng. Part A 2009, 15, 2977. 